\title{
LAS CONDICIONES PARA EL CONSENSO, LA VISIÓN DE CHARLES TAYLOR
}

\author{
Doris Elena Ospina Muñoz
Universidad de Antioquia- Colombia
}

\begin{abstract}
Resumen
Este artículo hace parte de una tesis doctoral inédita, titulada el consenso deseable, entre la visión de Charles Taylor y el cosmopolitismo contemporáneo. Basado en la lectura orientada al problema, el artículo introduce en las condiciones que, según la visión de Charles Taylor, requiere el consenso en las sociedades contemporáneas. Esas condiciones son: primero, la demostración de la tesis de la refutabilidad de las posiciones morales y, segundo, la ruptura con el etnocentrismo en el análisis de las situaciones de conflicto. La primera condición nos pone en contacto con las nociones básicas que estructuran el pensamiento de Taylor, a saber, autocomprensión, trasfondo moral compartido y orden moral secular. La segunda implica un marco de pensamiento político cosmopolita y producción de conocimiento transcultural que desafía el hacer de las ciencias sociales.
\end{abstract}

Palabras clave: consenso; etnocentrismo; cultura; visión moral; cosmopolitanismo.

Recibido: 07 de enero de 2016. Aprobado: 06 de diciembre de 2017. 


\title{
The Conditions in Order to Reach Consensus, the Charles Taylor's View
}

\begin{abstract}
This paper is part of a doctoral dissertation titled 'The desirable consensus, between Taylor's view and contemporary cosmopolitanism'. Through a systematic reading, the paper introduces the conditions in order to reach the consensus in contemporary societies. Such conditions are: first, a demonstration of the moral views' refutability thesis and, second, analyzing conflictive situations without ethnocentrism. First condition allows us to know about of Taylor's basic notions such as 'self-comprehension', 'shared moral background 'and 'secular moral order'. The second condition requires from us a frame for cosmopolitan thought and production of cross-cultural knowledge. These are challenges to social sciences
\end{abstract}

Keywords: consensus; ethnocentrism; culture; moral view; cosmopolitanism.

Doris Elena Ospina. Profesora titular, Universidad de Antioquia. Psicóloga (Universidad de Antioquia, 1994), Magister Salud Colectiva (Universidad de Antioquia, 1999), Especialista en investigación biomédica y psicosocial (Universidad de Chile, 2006), Magister en filosofía (Universidad de Antioquia, 2007), estudiante de doctorado en filosofía, Universidad de Antioquia, 2016). Sus áreas de trabajo y de investigación son: epistemología de las ciencias sociales, filosofía política, bioderecho y ética

Correo electrónico: doris.ospina@udea.edu.co 


\title{
LAS CONDICIONES PARA EL CONSENSO, LA VISIÓN DE CHARLES TAYLOR
}

\author{
Doris Elena Ospina Muñoz \\ Universidad de Antioquia- Colombia
}

\section{Introducción}

La pregunta sobre las posibilidades del consenso ha atravesado la historia de la filosofía política y de cierta manera ha sido, y será, la pregunta misma por la moralidad, en el sentido de aspirar a responder la cuestión de cómo hemos de vivir unos con otros, especialmente cuando estos otros no están cobijados por las mismas leyes. Así que aparezca en primer o segundo plano en la obra de un autor, el consenso es el tema en el que convergen la mayoría de las preocupaciones en los campos del pensamiento moral, político y social. En tiempos recientes, el debate sobre el consenso se ha concentrado alrededor de las críticas planteadas al liberalismo político de Rawls (1995), específicamente en lo relacionado al uso del constructivismo como método para la fundamentación teórica y a la psicología moral artificial que sostiene la fórmula del consenso traslapado.

En 1996 Taylor publicó un artículo que hace referencia explícita al tema del consenso: "A World Consensus on Human Rights?", reimpreso en 1999 y 2014 como "Conditions of an Unforced Consensus on Human Rights". Éste puede leerse como una crítica y una respuesta a la fórmula del consenso traslapado del liberalismo político de Rawls (1995). Lo primero que se observa en dicho artículo es la visión optimista de Taylor respecto a la posibilidad de alcanzar algún tipo de consenso, aún bajo la forma sugerida por Rawls, pero también señala algunas objeciones a esa fórmula. 
We would agree on the norms while disagreeing on why they were the right norms, and we would be contend in this consensus, undisturbed by the differences of profound underlying belief [...]. Is this kind of consensus possible? Perhaps because of my optimistic nature, I believe that it is. But [...] (Taylor, 2014c: 105).

Las objeciones giran alrededor de tres asuntos: sobre qué ha de formarse dicho consenso, su alcance, y cuáles son los obstáculos para lograrlo. El sobre qué ha de formarse el consenso es fácil de deducir a partir del título del artículo en mención, sobre los derechos humanos, pero no en la forma como ellos se leen en las cartas internacionales que forman el consabido "Bloque de Constitucionalidad" que aceptan la mayoría de los países democráticos en Occidente, sino sobre los valores que sustentan dichos derechos. Las razones para ello es que Taylor piensa que poner la mirada sobre los valores que sustentan los derechos humanos abre el alcance del posible consenso a sociedades no necesariamente democráticas, más o menos dispuestas a sostener un debate sobre derechos humanos. Centrar la atención sobre los valores y no sobre el contenido normativo que define los derechos en el ámbito internacional sería la garantía para romper con los mayores obstáculos que impiden, en opinión de Taylor, el avance del reconocimiento de los derechos humanos en el mundo: la atención a las formas occidentalizadas del lenguaje jurídico y el etnocentrismo en cuanto a la definición de sus contenidos. El supuesto de Taylor es que, aunque es probable que estos valores nunca puedan ser expresados en términos universales, dado que es presumible hallar restricciones entre las culturas para el mismo tipo de conductas, como por ejemplo, al genocidio, al asesinato, la tortura, la esclavitud, etc., es posible sostener que existen valores comunes profundos asociados a estas restricciones que deben ser develados.

Pero los argumentos de Taylor sobre el consenso no se construyeron para responder a la fórmula de Rawls, ellos hacen parte de una producción intelectual gestada desde los años 60 y que se actualiza, cuidando los efectos políticos, con argumentos de investigaciones provenientes de filosofía de la mente y el lenguaje.

En el contexto más amplio de la producción intelectual de Taylor se puede rastrear el siguiente argumento y plantearlo como una versión más completa y articulada de su idea sobre el consenso posible en las sociedades contemporáneas.

Los seres humanos se autocomprenden y comparten un trasfondo moral. En aras de alcanzar un consenso mundial voluntario sobre los valores pro- 
fundos que sustentan los derechos humanos, dicho trasfondo debe ser develado por medio de estudios sociales y políticos comparativos que ayuden a producir, mediante argumentación ad hominem, las transiciones morales necesarias para generar un nuevo orden moral. Este nuevo orden moral requiere mejores explicaciones y acomodaciones creativas que, entendidas como crecimiento moral en un tiempo secular, permitan reconocer y superar las diferencias y, en consecuencia, obtener el consenso ${ }^{1}$.

Esta formulación deja ver a Taylor como un perfeccionista moral, en el sentido de confiar en que el conocimiento producido, especialmente por las ciencias sociales y políticas puede ayudar a producir las transiciones morales necesarias para un nuevo orden moral. Así, al centro del planteamiento queda la argumentación ad hominem ${ }^{2}$, que entendida como forma de razonamiento práctico es la estrategia sugerida por Taylor para alcanzar el consenso. Pero dicha argumentación requiere dos condiciones: asumir como posible la refutación de la inconmensurabilidad de los juicios morales y la ruptura con el etnocentrismo, esto último es difícil de lograr por fuera de un marco de pensamiento político cosmopolita y sin producción de conocimiento transcultural.

\section{La refutación de la inconmensurabilidad o aceptación de la tesis de la corregibilidad de los juicios morales}

La argumentación ad hominem presupone la corregibilidad de las posturas morales. En Taylor existen dos vías para esta demostración. La primera vía parte de la discusión sobre la insuficiencia de los argumentos trascendentales para la justificación de posiciones morales, donde se asume

\footnotetext{
${ }^{1}$ Construcción personal a partir de la lectura de los textos del autor.

${ }^{2}$ Existe mucha controversia sobre lo qué son y lo que se puede lograr a partir de argumentos ad hominem. Según Taylor (1997) se trata de un tipo de argumentación profunda, un tipo de argumentación de la razón práctica capaz de presentar consideraciones (fines morales) con potencial para determinar al intelecto y, por tanto, susceptible de ser usadas para arbitrar entre posiciones rivales (p. 62). En la historia de la lógica y la retórica, el ad hominem (AAH) ha sido tradicionalmente considerado una falacia en tanto con frecuencia es difícil identificar una estructura que permita verlo como argumento y someterlo a algún criterio lógico y objetivo que soporte la crítica racional y evaluarlo como correcto o falaz. Sin embargo investigaciones recientes en lógica y argumentación han mostrado que los AAH tienen una estructura lógica que permite usarlos fácilmente como una sofisticada táctica despectiva contra lo mejor de un oponente o para atacar un argumento cuestionando la credibilidad del discutiente. Por esto cobran importancia en el campo de la pluralidad, donde se ha instalado la idea de que los juicios de valor son incontrovertibles. Igualmente, dichas investigaciones pretenden enseñar a usar y replicar los AHH de forma racional, preferencialmente, en ámbitos conversacionales y contextos apropiados de diálogo, en los que no pueden ser considerados falacias (Walton, 1998).
} 
que aunque las creencias morales deban, en principio, ser consideradas verdaderas, no son por ello necesariamente incontrovertibles. En cuanto creencias, las posiciones morales están sometidas a revisión constante ${ }^{3}$. Esta vía de argumentación hace parte de las críticas del autor al modelo epistemológico representacional y que desarrollé en otra parte ${ }^{4}$. La segunda vía implica, dada la profundidad del carácter moral como constitutivo de lo humano, una revisión de su vínculo con el concepto de libertad. No porque la libertad determine la moralidad sino por la imbricada relación entre ambas y sus efectos sobre la voluntad pues es sobre ésta sobre la que recaerá todo el peso de la vinculación de la voluntad a la norma, y por consiguiente, también el peso del consenso (Habermas, 1999). A continuación, en este artículo seguiré la argumentación de Taylor por esta vía.

No corresponde entrar aquí en las elevadas discusiones filosóficas que intentan explicar los vínculos entre las consabidas "condiciones de ser" (Lilli, 1949): el carácter moral, la voluntad, la libertad y el entendimiento. Nos conformaremos con la explicación racionalista que plantea que la voluntad es libre de orientarse al bien o al mal pero, a la vez, se deja permear por las razones que ofrece el entendimiento. Esta explicación cobra vitalidad en el mundo moderno, donde aquellas cuestiones, por ejemplo las creencias que orientan la acción, que estábamos destinados a aceptar como dictados de una autoridad externa (Dios, la naturaleza, la tradición) hemos de pensarlas por nosotros mismos como fruto de nuestra elección, convirtiendo la libertad y la autonomía en máximas de la acción moral (Taylor, 1994, p. 111).

Taylor (2005) plantea que el problema entre las dos concepciones de libertad es fundamentalmente un problema de articulación, por tanto su solución no es una concepción externa a los dos conceptos de libertad, sino precisamente una articulación entre ambos bajo la concepción de libertad como autorrealización, cuyo ejercicio exige autocomprensión; no existe libertad sin autocomprensión. El vínculo interno entre estos dos

\footnotetext{
${ }^{3}$ Esta afirmación se sostiene a partir de los debates sobre la insuficiencia de los argumentos trascendentales para justificar posiciones morales. Ver: Watt, A. J. (1975). Transcendental Arguments and Moral Principles. The Philosophical Quarterly, 25(98): 40-57. Disponible en: http://www.jstor.org/stable/2217951 [Acceso, abril 20 de 2015]. Walton, D. (1998). Ad hominem arguments. Studies in rhetoric and communication. The University Alabama Press. (1999). Informal Logic. A Handbook for Critical argumentation. Nueva York: Cambridge University Press(1999). Ethotic arguments and fallacies: The credibility function in multiagent dialogue Systems. Pragmatics \& Cognition 7(1), 177-203. Bruecker, A. (1996). Modest transcendental arguments. Philosophical Perspectives, 10, Metaphysics. Recuperado de URL: ttp://www.jstor.org/stable/i312591. DOI: 10.2307/2216247. Stable URL: http://www.jstor. org/stable/2216247

${ }^{4}$ Ospina D. Contexto y pensamiento de Charles Taylor sobre el consenso. (En proceso de publicación)
} 
hace insostenible la tesis de la incorregibilidad de las posturas morales que ha dominado el pensamiento liberal y que ha derivado en la fórmula de la neutralidad y el respeto indiferente a las nociones de vida buena.

Según Taylor, el concepto de libertad negativa nos dice que la libertad consiste en ser capaces de hacer lo que queramos, donde ese "lo que queramos" se entiende de manera no problemática como los deseos identificados por el agente en su carácter de tales (de meros deseos) (2005, p. 262). En contraste, Taylor sugiere una noción de libertad como ejercicio la cual exigiría discriminar entre motivaciones y considerar que los obstáculos a la libertad pueden ser tanto externos como internos: "Y es así porque las capacidades relevantes para la libertad deben implicar cierto grado de conciencia de sí, autocomprensión, discernimiento moral y autocontrol; de lo contrario, su ejercicio no podría equivaler a la libertad en el sentido de dirección de sí mismo" (2005, p. 263).

Como consecuencia de estas observaciones, la libertad como ejercicio no se conforma con la mera ausencia de obstáculos externos, precisa discernimiento sobre que lo que uno quiere no vaya en desmedro de sus objetivos fundamentales o su autorrealización. Exige discriminar entre motivaciones. El principal problema que esta concepción causa en el liberalismo es que se interpreta como una autorización para que otros, más conocedores de los objetivos del agente que él mismo, reorienten su marcha por el camino correcto, tal vez mediante el uso de la fuerza y todo ello en nombre de la libertad. Según Taylor (2005) se trata de una interpretación equívoca pues si se asume plenamente la idea de la libertad como autorrealización, es deducible que si bien pueden existir otros con un conocimiento íntimo de nosotros, que además nos superan en sabiduría y que están en condiciones de aconsejarnos, no existe ningún cuerpo oficial, dotado de una doctrina o una técnica que le permita saber cómo "encarrilarnos", porque esa doctrina y esa técnica no pueden existir por principio si los seres humanos difieren efectivamente en su manera de autorealizarse ( $p$. 264). Así, para Taylor es imposible defender cualquier noción de libertad que no implique algunas restricciones a la motivación entre las condiciones necesarias para la libertad y que excluya, por principio la posibilidad de que alguien se adelante al sujeto (p. 266). Es decir siempre cabe la pregunta sobre qué es lo correcto y lo incorrecto en términos de la libertad. A este argumento se suman tres ideas más: primero, que los obstáculos a la libertad se pueden jerarquizar dependiendo de la gravedad de la restricción que imponen a la libertad, eso es sobre lo que se discute en el ámbito público, político sobre lo que es significativo para la vida humana (p. 267). Segundo que esta jerarquización es posible porque existe un marco de comprensión que nos 
permite identificar que ciertas metas y actividades son más significativas que otras para la libertad. Dicho marco de comprensión es la identidad, que provee una distinción externa cualitativa para determinar si los deseos son elevados o bajos, nobles o viles, integrados o fragmentados, significativos o triviales, buenos o malos. Aquí la identidad se entiende "como el trasfondo en el que nuestros gustos y deseos, y opiniones y aspiraciones, cobran sentido" (1994, p. 70). Tercero, la identificación de los atribuidores de importancia para la libertad puede verse afectado por la influencia de la percepción y las emociones en la captación de las cosas; siempre existe el riesgo de que el sujeto se identifique con los deseos menos importantes en relación con la libertad, es decir que tenga una perspectiva distorsionada de ella. Por ejemplo, porque no reconoce las condiciones internas necesarias para la libertad o porque sus sentimientos confusos no le permitan juzgar adecuadamente lo verdaderamente importante en relación a lograr lo que realmente desea. Si se acepta eso como posible, entonces, en términos de la búsqueda de libertad, el sujeto puede estar equivocado, no puede ser el árbitro definitivo en este asunto, y la tesis de la incorregibilidad de los juicios de valor se hace insostenible.

[...] la libertad supone ahora, además de un camino libre de obstáculos externos, la capacidad de reconocer adecuadamente mis objetivos más importantes y de superar o al menos neutralizar mis impedimentos motivacionales. Pero es evidente que la primera condición (y yo sostendría que también la segunda) me exige haberme convertido en algo, haber alcanzado cierto estado de percepción y comprensión de mí mismo. Debo ejercer una verdadera autocomprensión para ser real o plenamente libre. Ya no puedo entender la libertad como un mero concepto de oportunidad (Taylor, 2005, p. 280).

Siguiendo con la argumentación de Taylor (2005), la autocomprensión es entonces una capacidad humana universal y una condición para el ejercicio de la libertad porque tiene el potencial para articular y transformar la experiencia. Esta idea recoge el carácter esencial, invariante e ineludible de la variabilidad humana: como seres lingüísticos, los humanos somos capaces de autointerpretación y autocomprensión.

I think we can say that being a linguistic animal is essential to one's having these concerns; because it is impossible to see how one could make a distinction like the one above, between, for example, things one just wants to do, and things that are worthy to be done, unless one was able to mark the distinction in some way: either by formulation in language, or at least 
by some expressive ceremonial which would acknowledge the higher demands (Taylor, 1985a. p. 103).

La tesis básica que la noción de autocomprensión encierra es que los seres humanos son animales que se autointerpretan. Es decir, los humanos pueden dar cuenta de sus experiencias de una manera que va más allá de ser meras descripciones subjetivas. "Our interpretation of ourselves and our experience is constitutive of what we are, and therefore cannot be considered as merely a view on reality, separable from reality, nor as an epiphenomenon, which can be by-passed in our understanding of reality" (1985b, p. 47).

Las autointerpretaciones de la experiencia son constitutivas del sujeto, pero no necesariamente correctas u objetivables en el sentido de obedecer a una descripción como la que se hace de los objetos. Tampoco son eternas. Por el poder articulador y creador del lenguaje, las autointerpretaciones quedan siempre sometidas a discusión, al encuentro de una mejor explicación, de tal manera que siempre será posible hallar nuevos significados para dichas experiencias. En ese someterse a discusión las experiencias se enfrentan a una especie de validación intersubjetiva (mediante el diálogo interno o externo) a la que podemos llamar autocomprensión.

Podemos ver que la noción de autocomprensión le permite a Taylor no quedarse en la dimensión básica del respecto que ha conducido al concepto de no intromisión o neutralidad sobre las concepciones de la vida buena. Él insiste en que estas posiciones son susceptibles de corrección y en que las elecciones deben ser hechas a la luz de los objetivos, pero también a la luz de una fuerte evaluación frente a lo que es deseable y valioso de ser deseado. Este tipo de evaluación resulta fundamental para el desarrollo de las capacidades humanas porque es a partir de poder elegir, distinguir y clarificar entre representaciones o deseos de primer y segundo orden desde donde podemos empezar a hablar de la agencia humana y la posibilidad de la construcción de un nuevo orden moral en donde los seres humanos, vistos como agentes sociales, deciden sobre lo bueno y lo realizable.

A partir de los expuesto hasta aquí podemos ver que la irrefutabilidad de las posiciones morales es insostenible siempre y cuando se acepte la existencia de un trasfondo moral compartido que ofrezca la posibilidad del contraste y la evaluación fuerte (Taylor, 2001). La idea del trasfondo moral compartido se sostiene, por un lado en la visión aristotélica del origen social de lo humano y, por el otro, en la idea iusnaturalista sobre elementos normativos comunes entre los pueblos. En la teoría de Taylor, la visión aristotélica se actualiza bajo la afirmación de que los seres humanos somos formados por el reconocimiento y que su falta o inadecuación genera daños 
profundos en la identidad (2001). También podemos decir que se materializa en el hecho de ser capaces de acoger al otro, de aceptar el intercambio y de construir un mundo común de convenciones o prácticas sociales (Taylor, 2006). Por su parte, la visión iusnaturalista supone la existencia de ideas compartidas de honradez y trato justo que pueden ser aceptadas por todos. Esta idea sirvió de base al desarrollo del derecho de gentes en el período en que Roma mantenía intercambio comercial con diversos pueblos. En la historia de la filosofía moral, estas ideas comunes tienen varias explicaciones: para algunos se trata de la legislación de los dioses que los gobernantes humanos no pueden alterar; para otros, se trata de dictados de la recta razón que se expresan como leyes eternas e inmutables aplicables a todos y que se explican dada la naturaleza social de los seres humanos, la cual lo llevaría no sólo a consentir las leyes sino también a entenderlas, todo ello con el fin de poder materializar su anhelo de compañía y vida en sociedad, justificación que se extiende con mayor razón a los casos en los que la convivencia se hace difícil (Schneewind, 2012).

Para Taylor, esta condición de sociabilidad obligada hace posible, mediante la persuación y en el marco de una política apropiada, argumentar razonablemente (ad hominem), en medio del relativismo blando que caracteriza el mundo moderno, sobre lo que sería un modo de vida mejor o superior para realizar el ideal de la autenticidad y la posibilidad de un nuevo orden moral (Taylor, 1994: pp. 67-110).

Los cambios o transformaciones en el orden moral son denominados por Taylor transiciones morales. Dichas transiciones aluden a un proceso complejo, que involucra tres ámbitos: el teórico, el de la autocomprensión y el normativo que se entrelazan más o menos así: ciertas prácticas discursivas se infiltran (parcialmente o por niveles) en la autocomprensión, lo cual, a su vez, se proyecta en el ámbito normativo, dando lugar a un orden moral nuevo que puede ser realizado o no. En el primero de los casos, estamos frente a una clave hermenéutica ${ }^{5}$ que da sentido a las prácticas sociales $\mathrm{y}$, en el segundo, frente a una prescripción normativa ${ }^{6}$, en algunas ocasiones utópica.

${ }^{5}$ Taylor usa el concepto de clave hermenéutica para referirse a una orientación discursiva que ha alcanzado suficiente reconocimiento en el medio social como para orientar y otorgar sentido a las prácticas individuales o colectivas dirigidas a un mejor ordenamiento social sin que esas orientaciones estén necesariamente reflejadas en normas de obligatorio cumplimiento.

${ }^{6}$ El concepto de prescripción normativa se refiere aquí a indicaciones o mandatos, generalmente establecidos jurídicamente o por códigos, de obligatorio cumplimiento, en beneficio del orden social. 
¿Qué quiere decir exactamente que una teoría penetre en un imaginario social y lo trasforme? En la mayoría de los casos, las personas asumen las nuevas prácticas por imposición, improvisación o adopción. A partir de este momento la práctica cobra sentido en virtud de la nueva perspectiva que ofrece, antes sólo articulada en la teoría; esta perspectiva es el contexto que da sentido a la práctica. La nueva idea aparece ante los participantes como nunca antes lo había hecho. Comienza a definir los contornos de su mundo y puede llegar a convertirse en el modo natural de ser de las cosas, demasiado evidente como para discutirlo siquiera. No se trata sin embargo de un proceso unidireccional, por el que una teoría construya su propio imaginario social. En el proceso de dar sentido a una cierta acción, la teoría se ve matizada en cierto sentido, toma una cierta forma como contexto de estas prácticas [...]. Tampoco tiene por qué terminar ahí el proceso. La nueva práctica, junto a la concepción de fondo generada por ella, puede servir de base para ulteriores modificaciones de la teoría, las cuales a su vez modificarán la práctica, y así sucesivamente (2006, p. 44).

Esto es lo que al parecer tiene lugar cuando Taylor sostiene que las teorías pueden impactar la mentalidad produciendo nuevas autocomprensiones que traen aparejado el concepto de crecimiento moral, referido a un proceso de ampliación de la mente (1997, p. 66), marcado a su vez por procesos históricos (Álvarez \& Paredes, 2002) 7 , y materializadas en innovaciones jurídicas y normativas; o lo que es lo mismo, en innovaciones tanto en las leyes como en las bases de fundamentación o justificación filosófica de las normas. Este proceso de crecimiento o maduración moral implica por un lado el descubrimiento, por deliberación, del orden moral correcto ${ }^{8}$ : las relaciones de beneficio mutuo que estamos destinados a realizar, y por el otro, el desarrollo del grado de autocontrol necesario para llevarlo a la práctica. Cuando avanzamos lo suficiente en ambos caminos llegamos al punto de inflexión, y es entonces cuando se abre la posibilidad de fundar una sociedad nueva y mejor.

En síntesis hasta aquí, tenemos que la primera condición para el consenso en la visión de Taylor, que he llamado la aceptación de la tesis de la corregibilidad de los juicios morales, requiere una revisión profunda del

\footnotetext{
${ }^{7}$ Que no es lo mismo que una revelación histórica de un plan oculto de la naturaleza como en Kant o en Hegel. Álvarez Gómez Mariano. "Kant y Hegel sobre el plan oculto de la naturaleza y de la historia”. En: Álvarez Gómez Mariano, Paredes Martín M ${ }^{\mathrm{a}}$ del Carmen. (Eds). La controversia de Hegel con Kant. II congreso Internacional. Sociedad Española de estudios sobre Hegel. Salamanca: Ediciones Universidad de Salamanca. 2002. pp. 85-98

${ }^{8} \mathrm{El}$ orden moral puede ser a nivel nacional, supranacional o incluso global debido a que un mismo imaginario social pueden permear todas las esferas. (Taylor 2006, pp. 205-212).
} 
concepto de libertad y dentro de ella de las nociones de autorrealización y autocomprensión. Pero, a su vez, la argumentación ad hominem requiere conocimientos y narraciones que determinen el intelecto, es decir que alcancen el punto de la mejor explicación posible en el marco de la discusión y la deliberación. La producción de nuevos conocimientos, que contribuyan a romper con el etnocentrismo, es una demanda de Taylor para las ciencias sociales y lo que he llamado la segunda condición para el consenso.

\section{Ruptura con el etnocentrismo}

El debate contra el etnocentrismo es más que una actitud de rechazo frente a aquellas interpretaciones que se muestran incapaces de abandonar la perspectiva del propio investigador o intérprete en el intento de comprender al otro. También contiene una profunda reflexión epistemológica sobre la aspiración de neutralidad que caracteriza al representacionalismo como modelo epistemológico dominante en el campo científico y sus efectos éticos y políticos. Esta reflexión constituye el segundo eje de la crítica de Taylor a dicho modelo. Desde ahí se muestran las complejas relaciones entre la forma como comprendemos el conocimiento y el ejercicio de la política.

Para Taylor (2014a), el "mito de la ilustración", es decir, aquella creencia de que las normas tienen que estar basadas en argumentos de la "sola razón", tiene su origen en dos movimientos. El primero es el del racionalismo cartesiano, el cual, a pesar de las críticas hechas por Hume a su excesiva confianza en las razones a priori y al desprecio por las entradas empíricas, fundó las creencias en la autonomía y autosuficiencia de la razón $\mathrm{y}$, en el potencial del método correcto para hallar la verdad. Prescindiendo de cualquier tipo de autoridad, en el modelo cartesiano solo se contó con lo que la razón monológica puede verificar como cierto. Según Taylor, el slogan de la época fue: "desafiar para conocer" (2014e, p. 325). Así, se llegó a considerar que las proposiciones racionales favorecían la continuidad hacia los juicios normativos, tal como han intentado demostrar tanto los utilitaristas como los kantianos, al sustentar, por ejemplo, que dado que los seres humanos son seres deseantes/disfrutantes/sufrientes o agentes racionales se puede seguir sin salto a la atribución de derechos. Para Taylor esta deducción es un principio de buena fe, tanto como aquel que afirma que la atribución de derechos se deriva del hecho de ser hijos de Dios y haber sido creados iguales ante sus ojos. De ahí que a Taylor le parezca infundada la distinción entre la credibilidad racional de los juicios basados en razones religiosas y los de la "sola razón".

${ }^{9}$ Hume D. (1983). Enquiry concerning the principles of morals: United States America: Hackett Publishing Company, Inc. ePub ISBN: 978-1-60384-713-1 
El segundo movimiento que dio origen al "mito de la Ilustración" fue la creencia de que los asuntos humanos y sociales pueden ser estudiados bajo el mismo modelo de las ciencias naturales. De este movimiento se heredó la creencia en la neutralidad de las ciencias, asunto que a Taylor también le parece un mito, que incluso puede ocultar la verdad al mismo autor (2014a, p. 328). Para Taylor (1997), el representacionalismo, con su idea del conocimiento como la correcta representación interna de una realidad independiente, está vinculado a nociones "muy influyentes y a menudo incompletamente formuladas sobre la ciencia y sobre la naturaleza de la capacidad de acción humana" (p. 21), y a través de ellas, "con ciertas ideas morales y espirituales centrales de la edad moderna" (p. 21). Una de estas nociones es la de certeza, entendida como algo que la mente debe generar, reflexivamente, por sí misma, para alcanzar la confianza bien fundada sobre la congruencia o correspondencia entre la representación interna y la realidad externa. Según Taylor, esta no es otra cosa que la correspondencia de una idea con la realidad. Adicionalmente, es el equivalente a los ideales morales de autorresponsabilidad y libertad como autonomía que se ilustran, por ejemplo, con las historias de cómo Copérnico y Galileo defendieron sus ideas. A pesar de las ideas de la época, ellos partieron de su propia certeza autorresponsable para defender y sustentar su pensamiento. Esto a su vez es congruente con la idea de que ser libre, en el sentido moderno, es ser autorresponsable, apoyarse en el propio juicio, hallar el propio propósito en sí mismo.

De esta manera Taylor (1997) muestra cómo la tradición epistemológica está enlazada con cierta noción de libertad y dignidad, desde donde la teoría del conocimiento y la libertad se refuerzan mutuamente, dando pie a algunas ideas (creencias) antropológicas acerca de la naturaleza del conocimiento humano. Estas son: primero, que el sujeto que conoce está idealmente desvinculado, es decir, es libre y racional hasta el punto de distinguirse totalmente de los mundos natural y social, de modo que su identidad ya no puede ser definida en términos de lo que está fuera de sí mismo, en estos mundos. Segundo, que el yo, en tanto libre y racional, es apto para tratar instrumentalmente con el mundo natural y social, e incluso el mundo interno, representado en algunos rasgos de su mismo carácter que pueden ser susceptibles de trasformación y reorganización en aras de mejorar y asegurar tanto el bienestar propio como el de los demás. Por último, como consecuencia de las nociones anteriores, la sociedad se entiende de forma atomista como constituida o explicable en términos de propósitos individuales. 
Estas nociones se concretan en visiones más amplias dentro de la cultura moderna: un sujeto alejado de su propio cuerpo, al que es capaz de observar como un objeto, lo cual pervive, más allá de la muerte del dualismo, en la exigencia contemporánea de una ciencia neutral y objetivadora de la vida y de la acción humanas[...]. Los ideales de gobierno y de reforma del yo [propios del siglo XVII perviven] en la tremenda fuerza que la razón instrumental y los modelos que la ingeniería tienen en nuestra política social, medicina, psiquiatría, política, etc.

[Todas ellas sobreviven] no solo en las teorías sucesoras del [contrato social], sino también en muchos supuestos del liberalismo contemporáneo y de la ciencia social dominante [...]. Superar o criticar estas ideas supone luchar a brazo partido con la epistemología. Y esto quiere decir entenderla en términos de lo que he identificado como su enfoque amplio, la entera interpretación representacional del conocimiento [...]. (Taylor, 1997, p. 27).

Estas reflexiones dan pie al llamado que Taylor hace a atender con mayor detenimiento el vínculo entre estudios factuales y creencias normativas; no porque considere que de los hechos se puedan deducir los valores, sino porque le parece una falacia sostener que los estudios científicos son neutrales y no ayudan a establecer o dar soporte a ninguna posición valorativa (1985c, pp. 58-90).

Las ciencias sociales que han mostrado cierta sensibilidad a esta afirmación, han aceptado que los valores interfieren en la producción de conocimiento, generando sesgos que pueden ser amortiguados tomando conciencia de ellos y reconociendo que tienen un papel activo durante la selección de las áreas de investigación y la interpretación de la información. De ahí que recomienden "poner afuera" los valores antes de comenzar un estudio y alertar al lector sobre su existencia. Pero, para Taylor, esto no asegura la neutralidad. Según él, esa es una tarea imposible pues al afirmar que una proposición es verdadera o falsa ya se le está cargando de valor.

Lo que a Taylor le parece más adecuado para sopesar este sesgo es socavar en la historia de los conceptos y sus acepciones de significado al momento de usarlos para el análisis de las situaciones que afectan la vida humana en sus diferentes ámbitos. En muchos casos los conceptos son usados para ayudar en el análisis de una situación dada desconociendo las raíces que dieron lugar a su significado, su estructura, su función y, en general, desconociendo los problemas que inicialmente intentó resolver. Así, la selección de conceptos podría explicar por qué un mismo hecho puede generar diferentes interpretaciones y juicios de valor.

Este problema de la neutralidad y la validez de los juicios de valor tiene un vínculo directo con el problema del reconocimiento de la igualdad, que 
como sabemos es de una importancia vital para el liberalismo. Cuando en "la política del reconocimiento" 10 , Taylor se alinea con la necesidad de una política de la diferencia para llegar a producir acuerdos en las sociedades multiculturales, que corrija las tendencias etnocéntricas, homogenizantes y de baja sensibilidad cultural que muestra el universalismo basado en la idea kantiana de la inherente dignidad humana (Giusti, 2014), está apelando a la presunción de igualdad de status ${ }^{11}$ para las culturas tradicionales, pero asume que dicha presunción es un desafío moral que tiene que demostrarse por encima de la propia voluntad y deseo, mediante el establecimiento de auténticos juicios de valor, no de aceptación por simple acomodación o condescendencia.

Con este planteamiento Taylor está desafiando la famosa fórmula de la neutralidad política entendida como igual respeto, la cual según Appiah (2007, p. 148), como mínimo, propende por evitar ofender las creencias de otros; considerar los intereses de la persona en juego durante una situación de conflicto entre sus creencias y las demandas del Estado y no tratar las creencias como simples preferencias. Esta es la fórmula que ha estabilizado, hasta ahora, el debate entre multiculturalitas sobre la pregunta ¿hasta qué punto es necesario el reconocimiento de las diferencias para sostener el respeto que se debe a los individuos? La concepción de la neutralidad como igual respeto es de uso corriente en los casos de confrontación jurídica y análisis de constitucionalidad.

Según Taylor, para el establecimiento de auténticos juicios de valor, y por tanto para el auténtico reconocimiento de la diferencia, requerimos ser transformados por el estudio del "otro", de modo que no solo juzguemos de acuerdo con nuestras normas familiares originales, sino mediante lo que Gadamer (2007) llama "fusión de horizontes". Dicha fusión solo es posible mediante la realización de estudios culturales comparativos, pues ellos desarrollan nuevos vocabularios de comparación que permiten expresar los contrastes entre culturas. De hecho, este tipo de estudios constituirían

${ }^{10}$ Un nuevo tipo de universalismo que, aunque anclado en la idea del respeto igualitario, apelaría más a los contenidos de la razón y a lo que se hace con ella (crear cultura) que a la razón misma para alcanzar el reconocimiento adecuado de la diferencia, asunto que se considera central en la construcción de la identidad moderna. Ver: Taylor Charles. El multiculturalismo y "la política del reconocimiento". Trad. Mónica Utrilla De Neira. México: Fondo de Cultura económica. 2001.

${ }^{11} \mathrm{El}$ reconocimiento de la igualdad de estatus es un presupuesto comunitarista que se basa en la idea de que para respetar a los individuos el Estado debe también hacer todo lo posible por respetar a las comunidades en la que ellos están insertos y las cuales dan contenido y contexto a sus opciones de vida. Ver Appiah Kwame Anthony. La ética de la identidad. Trad. Lilia Mosconi. Buenos Aires: Katz Editores. 2007. 
la base para demostrar la validez de otras culturas y establecer los criterios sobre lo cual han de considerarse valiosas. Con ellos se evitaría el riesgo etnocentrista de considerar como valioso solo aquellas cosas que son o parecen familiares o de las que se sospecha que podrían tener algún valor futuro en relación con los propios referentes, descuidando lo valioso que podría esconderse en lo que parece extraño y difícil.

Para Taylor (2014d) el modelo de la comprensión gadameriano rompe con el etnocentrismo porque evita el etiquetamiento de las prácticas y formas de vida de los extraños en relación con la vida propia, desde donde se hace imposible la creación de un punto de partida para la comprensión. Lo mismo ocurre con las explicaciones reduccionistas que son incapaces de establecer relaciones y contrastes. Por el contrario, una interpretación basada en asuntos comunes o similares abre el proceso de comprensión y este parece más esperanzador.

El punto importante es llegar a ver al otro correctamente. Eso significa alterar nuestra comprensión de nosotros mismos, involucrar un cambio en nuestra identidad, pues es la identidad por la que tan frecuentemente nos resistimos y rechazamos lo diferente. Tenemos una profunda identidad investida en la tergiversada imagen que nos hacemos de otros. Para obtener la distinción, nosotros tenemos que entender que afuera hay otras posibilidades, que nuestra manera de ser es solo una manera y representa una entre otras posibles formas. Así, la real comprensión siempre tiene un costo en la identidad (p. 37).

El costo aparece como ganancia una vez que ha sucedido el cambio: somos enriquecidos por el conocimiento de que otras posibilidades humanas existen en nuestro mundo. Esto no puede ser negado, sin embargo, ese camino al reconocimiento es frecuentemente doloroso. El momento crucial en este proceso es donde nosotros nos permitimos ser interpelados por los otros, donde las diferencias escapan desde su categorización como un error, una falta, algo insignificante o una subdesarrollada versión de lo que nosotros somos, y nos desafiamos a ver eso como una alternativa humana viable (Taylor, 2014d).

Como puede observarse, el modelo de comprensión gadameriano contiene una inevitable llamada a nuestra propia autocomprensión. A esto es lo que Gadamer (1993) llama "apertura" (p. 361). Dicha apertura no se trata del momento del inicio sino del momento donde yo intento reflexionar sobre mí mismo, sobre mi relación con los otros. Apertura a los otros, involucra reconocimiento de que yo mismo puedo aceptar algunas cosas negativas en mí. Este reconocimiento se asume como propio, nadie más me obliga a hacerlo (Taylor 2014d, p. 37). 
Según Taylor (2014d) el modelo gadameriano también representa una respuesta al relativismo. Siguiendo su exposición, para el relativismo las afirmaciones pueden ser juzgadas validas desde diferentes puntos de vista o perspectivas. La proposición $p$ podría ser verdad desde la perspectiva A, falsa desde la perspectiva $\mathrm{B}$, indeterminada desde $\mathrm{C}$, y así sucesivamente. Pero no habría tal cosa como su ser verdad o falsa incondicionalmente. Para Gadamer en cambio la diferencia entre perspectivas no es un asunto de validez o valores de verdad sino de las diferentes preguntas que pueden ser planteadas, de los diferentes problemas enraizados en cada una, de los diferentes rasgos que la hacen remarcable y así sucesivamente. Desde esta posición se puede entender que la ventaja de una sobre otra perspectiva radica en su capacidad para dar más y mejores respuestas, algunas de las cuales serán más correctas que otras o se acercarán a la verdad. Esto posibilita el ranking entre explicaciones bajo el criterio de ser más o menos comprensivas en un nuevo sentido del problema: no dependiendo de cuánto detalle o cobertura ofrecen del objeto estudiado sino, más bien, en su abordaje y forma de hacerlo comprensivo a una amplia banda de perspectivas. La más comprensible explicación en este sentido fusiona más horizontes (p. 32).

Mediante los estudios culturales comparativos se podría avanzar en la identificación de "tipos ideales" (no de categorías) que ayuden a identificar los modos de vida buena. En este asunto Taylor (1995) intenta recuperar la discusión sobre la imposibilidad de remover los valores o reducirlos a respuestas emocionales cuando se trata de hacer trabajo científico en ciencias humanas y sobre cómo los hallazgos científicos son relevantes para la formación de nuestros valores, en el sentido que ellos nos dirán cómo realizar las metas que nosotros nos ponemos a nosotros mismos. Adicionalmente, más allá de esto, Taylor quiere mostrar que las metas y los valores nos vienen de alguna parte más: de tradiciones que permanecen obscuras pero que pueden ser desveladas por medio de análisis históricos que muestren cómo determinados hechos afectaron a situaciones políticas y sociales e influyeron en la constitución de los mismos conceptos (Taylor, 1997).

La ruptura con el etnocentrismo implica también una ruptura con la creencia de que el pensamiento secular es de mayor envergadura epistémica que el pensamiento religioso. Esto es un asunto clave para entender el mundo contemporáneo con sus democracias diversas (Taylor, 2014d) y, en especial, a aquellas sociedades en las que los procesos de secularización han avanzado de manera diferente a la manera de Occidente. Esta distinción epistémica, entre pensamiento secular y pensamiento religioso, tiene un efecto directo sobre el trabajo de las ciencias sociales. Estas no pueden avanzar de la misma manera que las ciencias naturales porque no pueden ofrecer casos de 
contraste que permitan ver las ventajas de una teoría sobre otra. Las grandes teorías en ciencia social están separadas por su filosofía antropológica o su concepción de cómo los humanos se relacionan en sociedad, de las metas fundamentales de los propósitos humanos, de la distinción entre lo sagrado y lo profano, etc., por las explicaciones de ética y moral, y las virtudes de la sociedad. Por eso no pueden establecer conclusiones con la fuerza de principios ordenadores, porque el campo está dividido entre antropologías rivales, cada una de las cuales arroja su propia comprensión de los humanos en predicamentos socio-morales. La cuestión es que en este choque de visiones rivales, las antropologías mundanas no disfrutan de preferencia epistémica sobre las teológicas o religiosas. Así, la completa distinción entre visiones religiosas y entre razonamiento mundano resulta ser un espejismo y tenemos que reconocer que las ciencias sociales viven más por la fuerza sugestiva de sus narraciones (Taylor, 2014a).

A modo de conclusión, sin lugar a dudas, la invitación de Taylor (1997) a romper con las explicaciones neutrales en las ciencias sociales genera cierto escozor y nos pone frente a una encrucijada en relación con lo que conocemos y aceptamos como forma adecuada y correcta de producir conocimiento y hacer ciencia social; una ciencia social que a la vez que mejora las bases de su fundamentación, permita mejorar y desarrollar argumentos ad hominem en aras de alcanzar la mejor explicación posible.

Todo esto representa un desafío para la filosofía y las ciencias sociales. Implica un cambio de actitud y de métodos en la producción de conocimiento. El llamado parece orientarse al desarrollo de etnografías profundas (Appiah, 2007) o etnografías del valor (Courtney \& Taves, 2012), que permitan una revisión de la estructura de valores que da sentido a las diferentes formas contemporáneas de vida y contrastarla con la triada de valores de la Modernidad: libertad, igualdad y fraternidad. Quizás todos estos elementos requieren articulaciones disciplinares como las que señala Tugendhat (2007) entre antropología filosófica y cultural, a lo que Taylor, según Smith (2002), le sumaría una historia filosófica.

Este desafío a las ciencias sociales puede progresar por la vertiente del cosmopolitismo. Un concepto cargado de historia desde el mundo griego clásico y que en la contemporaneidad remite a las ideas de Kant, respecto a la posibilidad de que los individuos sean vistos como ciudadanos de un Estado universal de humanidad (Velasco, 1997). Ello, entendido como alcanzar un destino superior en cumplimiento del desarrollo de un plan oculto de la naturaleza humana: alcanzar un orden cada vez más racional y civilizado (Benhabib, 2011). Con dicho concepto no se indica una forma de organización política, sino una actitud ética hacia el mundo en la que 
todos los miembros de la raza humana, sin desvincularse de sus repúblicas, se unen en un orden civil para el ejercicio de la ciudadanía mundial, o la ciudadanía de la igualdad política (Redhead, 2002).

Según Castany (2006), a partir de la paz de Westfalia, en 1648, el discurso el cosmopolitismo dejó de ser compatible con el nuevo sistema sociopolítico, basado en la soberanía de los Estados-Nación y entró en una campaña de desprestigio y desfiguración que alcanzó a mostrarlo como un ideal demasiado abstracto, vago e ingenuo. Sin embargo, sostiene, que el concepto reapareció, a finales del siglo XX de manos de intelectuales como Ulrich Beck, Salman Rushdie, Martha Nussbaum, Juan Goytisolo o Edward. W., como una base muy importante de fundamentación de los derechos humanos, que amerita una recuperación semántica del concepto. En el marco de estudios culturales, filosóficos y de política internacional se usa el cosmopolitismo para referirse, en el contexto de las dinámicas globalizadoras, a la idea de un mundo poroso pero con suficientes cierres para mantener las ideas de soberanía, de derechos, de un orden racional que puede ser común a todas las ciudades humanas, y la creencia en la unidad de la humanidad a pesar de su diversidad (Benhabib, 2011).

Según Redhead (2002), quienes escriben en esta perspectiva, son autores eclécticos que comparten la idea de que la libertad para crearse a uno mismo necesita un amplio rango de opciones de sociabilidad transmitida, desde el cual se pueda inventar eso que hemos llamado identidad, sin requerir la glorificación de un vínculo particular con una cultura o la nación, pues cada persona adquiere o tiene una variedad de diferentes y dispares fidelidades o un estilo de vida híbrido, que es el único modelo saludable de subjetividad en tanto manifestación de la autonomía y la autenticidad. Esta concepción del cosmopolitismo no niega la idea de la identidad como constituida por diálogos morales intersubjetivos ni los desafíos metodológicos que ello representa para la ciencia social y el cuestionamiento moral (Benhabib, 2011).

A pesar de las críticas que Taylor recibe de los pensadores del cosmopolitismo es posible hallar varios puntos interesantes de articulación en especial lo referido a las concepciones de identidad y de cultura. Los cosmopolitanistas le cuestionan a Taylor que su pensamiento aún exhibe algunos rasgos nacionalistas y una concepción unitaria de las nociones de grupo y cultura que limitan la concepción de ciudadanía mundial. Estas críticas vienen principalmente de Seyla Benhabib (2006), con sus planteamientos sobre el cosmopolitismo normativo y de Appiah (2006), con sus reflexiones sobre la necesidad de una "teoría mixta de los valores" 
que revele los valores propios del cosmopolitismo y contraste con la tríada liberal de libertad, igualdad y fraternidad.

Para Benhabib (2011), por ejemplo, el cosmopolitismo, visto como actitud, rompe con las nociones unitarias de ciudadanía, grupo y cultura, propone una visión de estos conceptos como sistemas abiertos con contradicciones internas que ponen en tensión permanente la relación individuo-cultura-sociedad y la reivindicación de derechos humanos en todas las dimensiones de esa interacción. Benhabib (2011b) coincide con Taylor en estas cuestiones y en la idea de una democracia deliberativa que facilite "un diálogo cultural complejo", sin embargo, tiene ciertas reservas respecto al uso del concepto de reconocimiento tal como lo usa Taylor para referirse a las demandas de los movimientos actuales y sus corolarios en la política de la identidad y la diferencia. Ella considera, apoyada en resultados de estudios antropológicos que muestran cómo el reconocimiento político de los grupos bajo ciertas etiquetas limita la autonomía de los individuos dentro del grupo en cuanto a la construcción de sus proyectos de vida, lo cual parece especialmente cierto en los casos de las mujeres. Así, le parece a Benhabib que en el marco de las dinámicas internas de los grupos culturales, las reivindicaciones individuales de autoexpresión auténtica no tienen que ir de la mano de las aspiraciones colectivas de reconocimiento cultural, y que incluso pueden contradecirse entre sí. Encuentra un error teórico en la homologación de las reivindicaciones individuales y las colectivas facilitadas por las ambigüedades del término reconocimiento, lo cual, según ella, ameritaría una revisión, en el pensamiento de Taylor, de la conceptualización sobre grupo y cultura.

De igual manera Appiah (2006) concuerda con la necesidad de esta revisión y se aventura con la propuesta de un cosmopolitismo al que llama "con porvenir" (Appiah, 2007: 321), que articule lo que él llama el cosmopolitismo moral o universalista con la legitimidad, de al menos algunas formas de parcialidad o cosmopolitismo cultural. El primero, en términos generales, haría énfasis en los asuntos de ciudadanía compartida y las responsabilidades de unos con otros. Se trata de la vertiente que se corresponde con la idea de la existencia de una moral troncal común a la humanidad. El segundo, enfatiza en el compromiso de tomar seriamente, no solo el valor de la vida humana como tal sino también el valor de los modos particulares de vida; ello significa tomar interés en las prácticas y creencias que le dan significado a la vida. Ésta perspectiva pone en escena el lenguaje como vehículo de significación y engloba el primer tipo de cosmopolitismo, pues considera que cada forma de vida significativa habrá 
de responder, más allá de las preferencias particulares, a las obligaciones que tenemos con los demás.

El asunto problemático aquí, es que Appiah (2007) encuentra algo paradójico en la teoría normativa moderna: por un lado, apunta en el individualismo y en el carácter irreductible de la persona, pero por el otro, hace énfasis en la distinción entre miembros y no miembros, o entre quienes serán cobijados y quienes no por dichas normas facilitando el terreno para la discriminación. Al respecto dice: "Si todas las personas tienen el mismo valor, como afirman los liberales, ¿qué podría justificar que favorezcamos a los miembros de nuestro grupo respecto de los demás?" (p. 318). El llamado es a un discurso más profundo sobre la comprensión del otro, en el sentido de que "si nos interesamos por los otros que no hacen parte de nuestro orden político - los otros que pueden tener compromisos y creencias que son diferentes de las nuestras - debemos sentir una inclinación a hablar con ellos" (p. 320).

Según Appiah (2007), a lo largo de la historia en teoría política, cada una de estas perspectivas ha conducido por caminos diferentes. La primera, ha llevado necesariamente a la deducción de principios normativos, universalistas, para la orientación de la vida buena $-\mathrm{y}$ por tanto ha conducido a dinámicas de homogenización-, y la segunda, a la identificación, por medio de narrativas, de los valores centrales que constituyen una vida buena, en la que aún hay mucho que aprender, ya que hay tantas posibilidades humanas valiosas para explorar que resulta difícil pensar que todas las personas o todas las sociedades pueden converger en un modo singular de vida. Por eso su propuesta de realizar una teoría mixta de los valores que articule tanto principios dependientes de proyectos de vida como proyectos dependientes de objetivos más altos, lo cual, en cierto sentido recoge la crítica de Dworkin (1993) en cuanto a que no es posible seguir manteniendo la "esquizofrenia" o separación entre vida moral y política.

Appiah (2007) sugiere un método para el conocimiento de esos valores distintivos del cosmopolitismo que haga énfasis, en las distinciones entre: diversidad-divergencia y confrontación-conversación. Este método se llevaría a cabo mediante una práctica etnográfica densa, centrada no en la descripción de las diferencias, como hasta ahora ha sido, sino de las similitudes entre culturas, lo cual constituiría la base de los acuerdos.

Así las cosas, vemos que el cosmopolitismo más que refutar el pensamiento de Taylor lo amplía y, de alguna manera, pone a prueba la fuerza de sus argumentos básicos. En especial, que ni la cultura ni la identidad pueden concebirse como sistemas cerrados y que el reconocimiento de la diversidad de bienes y formas de vida buena no implica la negación de bienes 
constitutivos o ideales más altos que pueden, en medio de la diversidad, añadir sentido de trascendencia a la vida humana, haciéndola ver en conjunto como parte de un plan mayor de autorealización.

En síntesis, podemos decir que el cosmopolitismo recoge tres ideas que lo hacen coincidir con el pensamiento de Taylor. Primero, que el mundo es variado pero integrado en un orden; segundo, que en calidad de agentes morales todos estamos obligados a justificar nuestras acciones con razones frente a los demás o sus representantes; y tercero, que dada la obligación moral de justificación, todos los seres humanos, independientemente de sus circunstancias, participan de una conversación moral que trasciende los límites nacionales. Estas ideas han dado cuerpo a un proyecto filosófico normativo (Benhabib, 2011) y a una agenda de investigación en ciencias sociales (Fine 2007), que pone al centro el concepto de reconocimiento y, en ese sentido, plantea una discusión con Taylor.

El proyecto filosófico normativo del cosmopolitismo es definido por Benhabib (2011) como un proyecto de mediaciones o emergencia de normas (jurídicas y morales) en una sociedad civil global, que busca extender los principios universalistas de la ética del discurso para la validez de las normas más allá de los confines de la Nación-Estado. En esta línea, el debate con Taylor se centra, primero, en que él no busca principios únicos, como por ejemplo, el principio del discurso habermasiano, para resolver los conflictos, sin embargo es defensor de una política deliberativa y dialógica (del caso por caso), y, como vimos, defiende la argumentación persuasiva como vía para el consenso; segundo, en que Benhabib, apoyada en estudios antropológicos, duda sobre la capacidad del concepto de reconocimiento tayloriano para proteger los derechos individuales dentro del grupo. Esto último encaja con la resistencia de los liberales a considerar que los derechos individuales requieren ser protegidos tanto en la instancia colectiva como en la individual.

La crítica liberal a la defensa de los derechos colectivos olvida que en Taylor el llamado es a lograr el pleno reconocimiento y el tratamiento de la gente a partir de una política de la diferencia que evalue y justifique las diferentes situaciones $y$, en consecuencia, responda de forma más adecuada a sus demandas de reconocimiento, las cuales, en algunos casos, obedecen a demandas en el plano de la identidad individual y otras a la identidad compartida. Esta orientación al reconocimiento de la diferencia está fundamentada en que tanto los rasgos individuales como los colectivos son relevantes para la identidad y generan diferentes requerimientos, con lo cual, en consecuencia, necesitan diferentes tipos de respuesta, ya sean personales, institucionales, y/o sociales. Dado que muchas de estas respuestas resultarán controversiales se requiere un marco normativo democrático deliberativo en 
el que los asuntos de sensibilidad pública pueden ser discutidos de forma ampliada (Laitinen, 2012).

El cosmopolitismo, en cuanto una agenda de investigación en ciencias sociales, según Fine (2007), tiene ante todo un carácter crítico y ha asumido principalmente tres compromisos: reflexionar sobre el nacionalismo metodológico y su influencia en la generación de conocimiento en las ciencias sociales, ampliar la comprensión del cosmopolitismo más allá de la concepción kantiana de hospitalidad y explorar las posibilidades de la primacía de una ciudadanía mundial sobre cualquier filiación particular (nacional, étnica, religiosa, cultural, etc.). En ese sentido hay una coherencia con la demanda de Taylor sobre la necesidad de romper con la tradicional forma de hacer conocimiento social. En esta línea, los llamados de Appiah (2007) en cuanto a volver a pensar conceptos tales como los de identidad y cultura, y reflexionar sobre las posibilidades del ideario valórico del liberalismo en las sociedades contemporáneas tienen pertinencia.

Las preguntas y tensiones que pueden ser abordadas por las ciencias sociales hermenéuticas pueden ser las mismas que hasta ahora han asumido los estudios sociales empíricos comparativistas, pero la diferencia radical entre ambos enfoques estaría en la relación que establecen con los datos. Por ejemplo, etnografías que han intentado responder a preguntas sobre cómo la gente está definiendo lo que considera importante en un tiempo secular, qué fines persiguen, cuáles son las cosas que más valoran para la definición de la vida buena, cómo se da el proceso de evaluación y valoración de las cosas que tiene valor, cómo se dan los procesos de toma de decisiones, han encontrado que estos asuntos se están resolviendo en un amplio espectro de eventos y organizaciones políticas e institucionales que confluyen en la formación de historias locales, trayectorias y transiciones empíricamente observables. El análisis empírico de estos fenómenos muestra prácticas y acciones, tales como cambios en las leyes, interacciones sociales y acuerdos económicos, estructuras y demandas jurídicas y la acción de los movimientos sociales en la redefinición de las prácticas que encarnan lo secular (Courtney \& Tave, 2012). Sin embargo, para Taylor, dichos fenómenos ameritan acercamientos hermenéuticos, pues de lo contrario no se logrará pasar del primer nivel del círculo hermenéutico: la descripción de los "datos brutos", dejando en una especie de limbo la riqueza fenomenológica y afectiva de lo que significa habitar un tiempo secular y avanzar hacia niveles superiores de crecimiento moral (Smith J, 2014).

Del modo empírico se pierde la oportunidad de alcanzar las significaciones intersubjetivas y comunes con potencial normativo para fundamentar, por ejemplo, el consenso. Este es el desafío que enfrentan las 
ciencias hermenéuticas; para que puedan cumplir ese desafío dichas ciencias tiene que enfrentar varias dificultades, la primera tiene que ver con preguntas de orden ontológico, referidas al objeto de estudio, y las segundas son de orden metodológico previas a la aplicación de las fases de la reducción fenomelógica. Ambas dificultades se deben a que las significaciones intersubjetivas y comunes, se forman y viajan a través del lenguaje. De ahí que, más que tener que lidiar con las creencias y valores, lo que desafía a las ciencias hermenéuticas a romper con el modelo epistemológico tradicional es la apelación al lenguaje como vía de objetivación de las significaciones. Resolver estos problemas es una tarea urgente para consolidar el salto epistemológico que requieren las ciencias sociales hermenéuticas (Catoggio, 2008). 


\section{Referencias bibliográficas}

Álvarez, M. (2002). "Kant y Hegel. Sobre el plan oculto de la naturaleza y de la historia". En: Álvarez Gómez Mariano, Paredes Martín Ma del Carmen. (Eds). La controversia de Hegel con Kant (pp. 85-98). II Congreso Internacional. Sociedad Española de estudios sobre Hegel. Salamanca: Ediciones Universidad de Salamanca.

Appiah, K.A. (2006). Cosmopolitanism. Ethics in a world of strangers. New York: W.W Norton \& Company. (Kindle edition)

Benhabib, S. (2006). Another Cosmopolitanism. Oxford: University Press. (Kindle edition).

Benhabib, S. (2011a). Las reivindicaciones de la cultura. Igualdad y diversidad en la era global. Trad. Alejandra Vasalla. Buenos Aires: Katz. Discusiones. (Kindle edition).

Benhabib, S. (2011b). Dignity in adversity. Human rights in turbulent times. Cambridge: Polity Press. (Kindle edition).

Castany, B. (2006). Apología del cosmopolitismo. Konvergencias, Filosofia y Culturas en Diálogo. [Online]. Disponible en: http://www.konvergencias.net/ castanyprado113.htm [acceso, agosto 26 de 2015].

Catoggio, L. (2008). "Límites y alcances de la fusión de horizontes en la comprensión intercultural". En: Revista de filosofia (Universidad Iberoamericana), 121,65-82

Courtney, B. \& Taves, A. (Ed). (2012). What Matters? Ethnographies of value in a not so secular age. New York: Columbia University Press (Kindle edition). E- ISBN 978-0-231-50468-3.

Dworkin, R. (1985). A matter of principle. Cambridge. Harvard University Press.

Dworkin, R. (1993). Ética privada e igualitarismo político. Barcelona: Paidós.

Dworkin, R. (2013). "El lugar de la libertad". En: Virtud Soberana. La teoría y la práctica de la igualdad. España: Paidós. pp. 133-201.

Fine R. (2007). "Cosmolitismo: una agenda de investigación para las ciencias sociales". En: Kozlarek Oliver (Comp). Entre Cosmopolitismo y conciencia del mundo. Hacia una crítica del pensamiento atópico (pp. 16-36). México: Siglo XXI. CREFAL.

Gadamer, G. (1993). Verdad y Método I. Fundamentos de una hermenéutica. Trad. Ana. Agud Aparicio y Rafael de Agapito. Salamanca: Ediciones sígueme.

Gadamer, G. (1998). Verdad y Método II. Trad. Manuel Olasagasti. Recuperado de: Disponible en URL: http://www.olimon.org/uan/gadamer-verdad_y_metodo_ ii.pdf

Gadamer, G. (2007). El problema de la conciencia histórica. Trad. Agustín Domingo Moratalla. 3ra Ed. Madrid: Tecnos.

Giusti, M. (1996). "Paradojas recurrentes de la argumentación comunitarista". En: Cortés.

Rodas Francisco y Monsalve Solórzano Alfonso. Liberalismo y comunitarismo. Derechos humanos y democracia (pp. 99-126). España: Edicions Alfons el Magnànim - IVEI. 
Rodas Francisco y Monsalve Solórzano Alfonso. (2014). Los derechos humanos en un contexto intercultural. Organización de Estados Iberoamericanos para la educación, la ciencia y la cultura. Disponible en: http://www.oei.es/valores2/ giusti2.htm [acceso: marzo 14 de 2014].

Habermas, J. (1999). “Una consideración genealógica acerca del contenido cognitivo de la moral". En: La inclusión del otro (pp. 29-18). Barcelona: Paidós.

Laitinen, A. (2012). "Misrecognition, Misrecognition, and Fallibility". Res Pública, 18(1), 25-38.

Lilli F. (1949). Libre yo y libertad moral. Actas del Primer Congreso Nacional de Filosofía, Mendoza, Argentina, marzo-abril 1949, tomo 2. [online] Disponible en: http://www.filosofia.org/aut/003/m49a1305.pdf [Acceso, agosto 19 de 2015]

Rawls, J. (1995). Liberalismo político. México: Facultad de Derecho UNAM; Fondo de Cultura Económica. Recuperado de: http://dialnet.unirioja.es/servlet/ articulo?codigo $=241247$ [Acceso: abril 12 2015].

Redhead, M. (2002). Charles Taylor. Thinking and living Deep Diversity. United States Of America. Rowman \& Littlefield publishers, Inc. (Kindle edition).

Schneewind J.B. (2012). La invención de la autonomía. Una historia de la filosofia moral moderna. Trad. Jesús Héctor Ruíz Rivas. México: Fondo de Cultura Económica. (Kindle edition). ISBN; 978-607-16-1143-7.

Smith, J.K.A. (2014). How (not) to be secular. Reading Charles Taylor. Michigan/ Cambridge: William B. Eerdmans Publishing Company. ePub Kindle: eISBN 978-1-4674-4019-6.

Smith, N.H. (2002). Charles Taylor. Meaning, Morals and Modernity. Cambridge: Polity Press. (Kindle edition) ISBN: 9780745668598

Taylor, Ch. (1985a). "The concept a person". En: Human Agency and Language. Philosophical Papers I (pp. 97-114). Cambridge University Press.

Taylor, Ch. (1985b). "Self-interpreting animals". En: Human Agency and Language. Philosophical Papers I (pp.45-76). Cambridge University Press.

Taylor, Ch. (1985c). "Neutrality in Political Science". En: Philosophy and the Human Sciences: Philosophical Papers 2 (pp. 58-90). Cambridge: Cambridge University Press.

Taylor, Ch. (1994). La ética de la Autenticidad. Barcelona, España: Paidós.

Taylor, Ch. (1995). "Comparison, History, Truth”. In: Philosophical Arguments (pp.146-165). Cambridge, MA: Harvard University Press.

Taylor, Ch. (1997). Argumentos filosóficos. Ensayos sobre el conocimiento el lenguaje y la Modernidad. Trad. Fina Birulés Bertrán. Barcelona: Paidós.

Taylor, Ch. (2001). El multiculturalismo y "la política del reconocimiento". Trad. Mónica Utrilla De Neira. México: Fondo de Cultura económica.

Taylor, Ch. (2005). “¿Cuál es el problema de la libertad negativa?”. En: La libertad de los modernos (pp. 257-281). Trad Horacio Pons. Buenos Aires: Amorrortu.

Taylor, Ch. (2006). Imaginarios sociales modernos. Trad. Ramón Vilá Vernis. Barcelona: Paidós.

Taylor, Ch. (2007). La ética de la identidad. Trad Lilia Mosconi. Buenos Aires: Katz Editores. 
Taylor, Ch. (2014). Dilemmas and connections. Selected essays. United States of America: First Harvard University Press paperback edition.

Taylor, Ch. (2014a). Die Blosse Vernunft ("Reason Alone"). Dilemmas and connections. Selected essays. United States of America: First Harvard University Press paperback edition. pp.326-346

Taylor, Ch. (2014b) "Perils of moralism" (pp. 347-366). En: Dilemmas and connections. Selected essays. United States of America: First Harvard University Press paperback edition.

Taylor, Ch. (2014c). "Conditions of an unforced consensus on human rights" (pp. 105-123). En: Dilemmas and connections. Selected essays. United States of America: First Harvard University Press paperback edition.

Taylor, Ch. (2014d). "Understanding the Other: A Gadamerian View on Conceptual Schemes" (pp.24-38). En: Dilemmas and connections. Selected essays. Cambridge, MA: Harvard University Press.

Taylor, Ch. (2014e). "What does secularism mean?" (pp.303-325). En: Dilemmas and connections. Selected essays. United States of America: First Harvard University Press paperback edition.

Tugendhat, E. (1996). "Identidad Personal, Nacional y Universal". Revista Ideas $y$ Valores. 100. Bogotá.

Tugendhat, E. (2001). “Moral desde la teoría de la evolución”. En: Diálogo científico (Tubingen). 10: 01-02. 11-34.

Tugendhat, E. (2007). “Antropología como filosofía primera". Thémata. Revista de filosofia, (39), 41-47.

Velasco Arroyo, J.C. (1997). "Ayer y hoy del cosmopolitismo kantiano". Isegoría, $16,91-117$. 